\title{
DNA Transposon Expansion is Associated with Genome Size Increase in Mudminnows
}

\author{
Robert Lehmann $\mathbb{D}^{1}$, Aleš Kovařík ${ }^{2}$, Konrad Ocalewicz ${ }^{3}$, Lech Kirtiklis ${ }^{4}$, Andrea Zuccolo ${ }^{5,6}$, Jesper N. Tegner ${ }^{1}$,

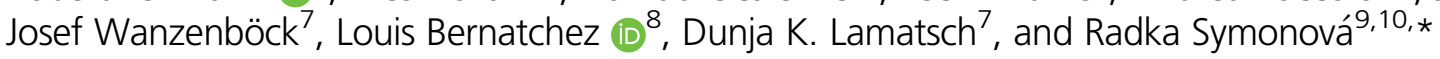 \\ ${ }^{1}$ Division of Biological and Environmental Sciences \& Engineering, Computer, Electrical and Mathematical Sciences and Engineering Division, King \\ Abdullah University of Science and Technology, Thuwal, Kingdom of Saudi Arabia \\ ${ }^{2}$ Laboratory of Molecular Epigenetics, Institute of Biophysics, Czech Academy of Science, Brno, Czech Republic \\ ${ }^{3}$ Department of Marine Biology and Ecology, Institute of Oceanography, Faculty of Oceanography and Geography, University of Gdansk, Gdansk, \\ Poland \\ ${ }^{4}$ Department of Zoology, Faculty of Biology and Biotechnology, University of Warmia and Mazury, Olsztyn, Poland \\ ${ }^{5}$ Center for Desert Agriculture, Biological and Environmental Sciences \& Engineering Division (BESE), King Abdullah University of Science and \\ Technology, Thuwal, Kingdom of Saudi Arabia \\ ${ }^{6}$ Institute of Life Sciences, Scuola Superiore Sant'Anna, Pisa, Italy \\ ${ }^{7}$ Research Department for Limnology Mondsee, University of Innsbruck, Mondsee, Austria \\ ${ }^{8}$ Department of Biology, IBIS (Institut de Biologie Intégrative et des Systèmes), Université Laval, Québec, QC, Canada \\ ${ }^{9}$ Department of Bioinformatics, Wissenschaftzentrum Weihenstephan, Technische Universität München, Freising, Germany \\ ${ }^{10}$ Department of Biology, Faculty of Biology, University of Hradec Kralove, Czech Republic \\ *Corresponding author: E-mail: radka.symonova@gmail.com.
}

Accepted: 27 September 2021

\begin{abstract}
Genome sizes of eukaryotic organisms vary substantially, with whole-genome duplications (WGD) and transposable element expansion acting as main drivers for rapid genome size increase. The two North American mudminnows, Umbra limi and Umbra pygmaea, feature genomes about twice the size of their sister lineage Esocidae (e.g., pikes and pickerels). However, it is unknown whether all Umbra species share this genome expansion and which causal mechanisms drive this expansion. Using flow cytometry, we find that the genome of the European mudminnow is expanded similarly to both North American species, ranging between 4.5 and $5.4 \mathrm{pg}$ per diploid nucleus. Observed blocks of interstitially located telomeric repeats in U. limi suggest frequent Robertsonian rearrangements in its history. Comparative analyses of transcriptome and genome assemblies show that the genome expansion in Umbra is driven by the expansion of DNA transposon and unclassified repeat sequences without WGD. Furthermore, we find a substantial ongoing expansion of repeat sequences in the Alaska blackfish Dallia pectoralis, the closest relative to the family Umbridae, which might mark the beginning of a similar genome expansion. Our study suggests that the genome expansion in mudminnows, driven mainly by transposon expansion, but not WGD, occurred before the separation into the American and European lineage.
\end{abstract}

Key words: genome expansion, Umbra, Robertsonian fusion, centric fission, repetitive sequences.

\section{Introduction}

The driving forces and effects of genome size variations across different taxa are a recurring theme in the field of evolutionary biology (Lynch 2007). While the number of chromosomes is typically highly conserved among fishes (Mank and Avise 2006), genome sizes vary substantially and include the smallest and the largest vertebrate genome (Hardie and Hebert 2004). Specifically, fish genome size ranges from

(C) The Author(s) 2021. Published by Oxford University Press on behalf of the Society for Molecular Biology and Evolution.

This is an Open Access article distributed under the terms of the Creative Commons Attribution License (https://creativecommons.org/icenses/by/4.0/), which permits unrestricted reuse, distribution, and reproduction in any medium, provided the original work is properly cited. 


\section{Significance}

North American mudminnows feature genomes about twice the size of their sister lineage Esocidae (e.g., pikes and pickerels). However, neither the mechanism underlying this genome expansion, nor whether this feature is shared amongst all mudminnows is currently known. Using cytogenetic analyses, we find that the genome of the European mudminnow also expanded and that extensive chromosome fusion events have occurred in some Umbra species. Furthermore, comparative genomics based on de novo assembled transcriptomes and genome assemblies, which have recently become available, indicates that DNA transposon activity is responsible for this expansion.

$C=0.35 \mathrm{pg}$ in bandtail pufferfish (Sphoeroides spengleri) to $\mathrm{C}=133 \mathrm{pg}$ in marbled lungfish (Protopterus aethiopicus) (Gregory 2020). The main candidate processes introducing such drastic variation are gene (Lu et al. 2012; Ohno 2013) or genome duplication (Fontdevila 2011; Van de Peer et al. 2017), transposable element (TE) proliferation (Pritham 2009; Tenaillon et al. 2010), and replication slippage at tandem repeat loci (Ellegren 2004). Furthermore, it is unclear whether this variation is shaped by adaptive processes (Gregory and Hebert 1999; Liedtke et al. 2018; Van de Peer et al. 2017) or stochastic sequence gain and loss (Lynch 2007). The presence of TEs in virtually all eukaryotic genomes suggests a role of stochastic sequence gain due to TE proliferation (Elliott and Gregory 2015). There are several recent reports of significant genomic expansion, such as in salamanders due to long terminal repeat element activity (Sun et al. 2012) and in Hydra due to long interspersed nuclear element activity (Wong et al. 2019). Estimates of the pervasiveness and extent of expansion events caused by TE proliferation and other processes will provide insight into the forces that shape genome size evolution.

Mudminnows (Umbridae) are very resilient members of the order Esociformes, capable of withstanding extreme cold and can utilize atmospheric oxygen. Under adverse conditions, they are reputed to become dormant in mud (Gilbert and Williams 2002). The order Esociformes includes two families and four genera in at least 12 species with one fossil-only family (Nelson et al. 2016). Furthermore, Esociformes (Haplomi, Esocae; pikes and mudminnows) and its sister order Salmoniformes belong to the clade Protacanthopterygii, a lineage of basal teleosts (Nelson et al. 2016). Genome sizes of both North American Umbra species were determined already in the 1960 s to be $C=2.52-2.70 \mathrm{pg}$ for Umbra limi and $C=2.4 \mathrm{pg}$ for Umbra pygmaea (Hinegardner 1968). The genome sizes of the remaining representatives of the order Esociformes, including Esox, Novumbra, and Dallia, ranges from 0.85 (in Esox) to 1.4 (also in Esox; summarized by (Gregory 2020); details in supplementary table S1, Supplementary Material online). While two of the three extant Umbra species feature a genome size twice the size of the remaining esociform species, it is currently not known whether this is a universal feature of the Umbridae and can be expected in Umbra krameri.
The diploid chromosome number in Esociformes ranges from $2 n=22$ (U. pygmaea and $U$. limi) to $2 n=71-79$ (Dallia sp.). Almost all known pike (Esox) species possess 50 (fundamental number $\mathrm{FN}=50$ ) usually acrocentric chromosomes, including species from Canada, USA, Sweden, and Russia (Arai 2011) and two European Esox species (Symonová et al. 2017). This karyotype is considered the preduplication ancestor of the order Salmoniformes (salmon, grayling, whitefish) (Rondeau et al. 2014; Ráb 2004). There are, however, reports of $\mathrm{FN}=86$ with $2 n=50$ in Esox lucius from the south Caspian Sea basin and further reports of FN = 72-88 in other E. lucius populations (Khoshkholgh et al. 2015). This morphological variability of chromosomes in the Esox genus with its broad circumpolar distribution (Nelson et al. 2016) has not yet been analyzed.

Pikes are highly interesting from the cytogenomic viewpoint because of their extremely amplified, massively methylated, and potentially functional $5 \mathrm{~S}$ rDNA recorded on more than 30 centromeric sites of their 50 chromosomes, whereas $45 \mathrm{~S}$ rDNA is located in only two sites (Symonová et al. 2017). A similar amplification and increased dynamics of the $45 \mathrm{~S}$ rDNA fraction has been repeatedly recorded in Salmoniformes (Gregory and Hebert 1999; Liedtke et al. 2018), which furthermore underwent an already wellcharacterized whole-genome duplication (WGD) (Macqueen and Johnston 2014; Lien et al. 2016). Indeed, WGD events have been described for multiple basal, particularly freshwater teleosts such as Cypriniformes (Li et al. 2015) and Siluriformes (Marburger et al. 2018). This observation suggests two distinct explanations for the significant genome size increase of Umbridae compared to its sister lineage Esocidae, an Umbridae-specific WGD on the one hand, and an extreme amplification of ribosomal DNA on the other.

Here, we present cytogenomic evidence that all extant members of the Umbra lineage feature an expanded genome size compared to its closest relative, the Esocidae family. Furthermore, we find that Umbridae feature a standard locus and copy number of $5 S$ rDNA typical for teleosts, excluding a $5 S$ rDNA expansion as a reason for the increased Umbra-specific genome size. While phylogenetic profiles of orthologous genes across species do not show patterns of WGD, comparative analyses of repetitive sequence content across six 

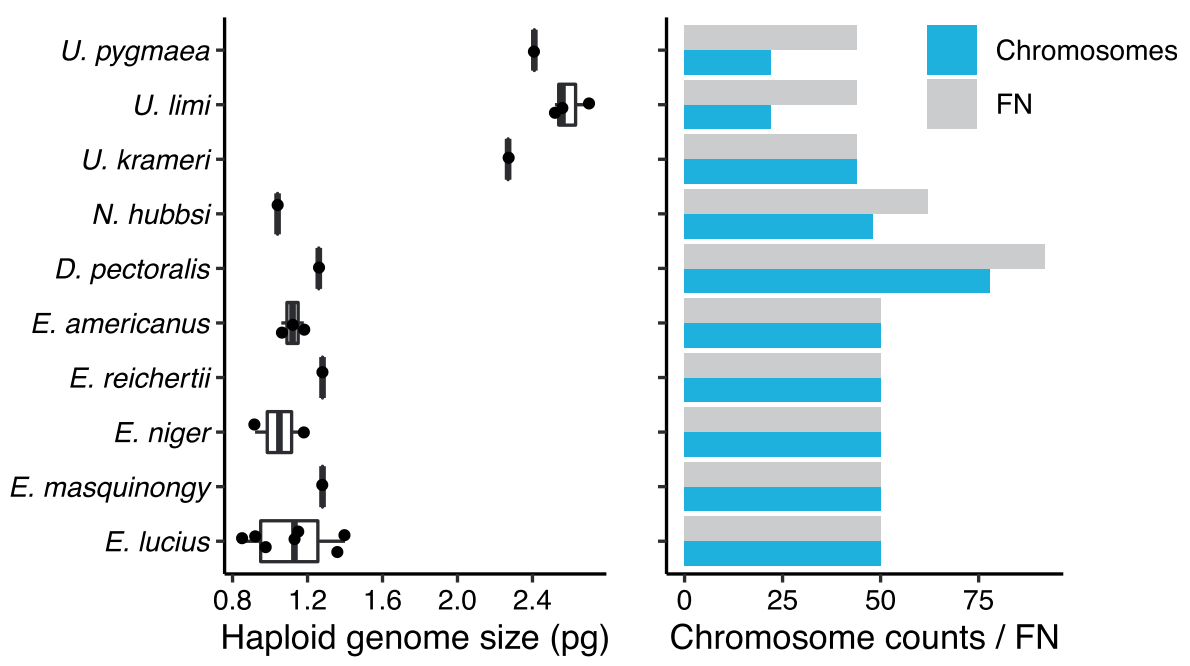

Fig. 1.-Genome size (C-value) (left) and chromosome (2n) and chromosome arms (FN) counts (right) in esociform fish.

Esocidae species show a clear signal of DNA transposon expansion in U. pygmaea driving the genome size increase.

\section{Results \\ Flow Cytometry Genome Size Determination in the European Pike and the European Mudminnow}

Flow cytometry measurements using DAPI staining of $U$. krameri with chicken RBC as internal standard exhibited a genome size of $2 \mathrm{C}=4.54 \pm 0.05 \mathrm{pg}( \pm$ STDEV) per nucleus (CV $\% 3.36 \pm 0.55$; range $4.43-4.60 \mathrm{pg}$ per nucleus, $N=10$ ). Esox lucius gave genome values of $2 \mathrm{C}=2.22 \pm 0.03 \mathrm{pg}$ per nucleus (CV \% 2.44 \pm 1.37 , range 2.18-2.27 pg per nucleus, $N=2$ ). Genome size and chromosome traits ( $2 n$ and FN) for the order Esociformes are summarized in figure 1 and show the elevated genome size in all three Umbra species. The higher interquartile range in $E$. lucius reflects merely the higher sampling effort and multiple values available. A similar situation exists in $U$. limi. We provide an overview of cytogenomic and cytogenetic traits of the order Esociformes (supplementary table S1, Supplementary Material online) based on the checklist of fish karyotypes (Arai 2011) and other literature records and online resources.

\section{Comparative Analysis of the U. pygmaea Transcriptome Does Not Indicate Whole Genome Duplication}

To test whether the Umbridae family underwent a wholegenome duplication event (WGD), we assessed gene duplication levels in a set of 24 de novo transcriptome assemblies, including the eastern mudminnow U. pygmaea (Pasquier et al. 2016). We focused on a set of 3,640 benchmark universal single-copy orthologous (BUSCO) genes, which occur only once in the majority (>90\%) of a set of 26 selected Actinopterygii species (Simão et al. 2015). Since only a single salmonid species, Salmo salar, was used to construct the
BUSCO set, genes that were duplicated in the salmonidspecific WGD (Macqueen and Johnston 2014) were not excluded. Similarly, as this gene set does not consider Umbridae family species, any potential ohnologs originating from an Umbridae-specific WGD are not excluded. Accordingly, five of the six salmonid de novo transcriptome assemblies show the highest duplication level amongst the 24 considered species (supplementary fig. S2 and table S2, Supplementary Material online). In contrast, U. pygmaea exhibits the second-lowest duplication level, second only to Pangasius hypophthalmus. The elevated duplication level in European eel Anguilla anguilla and arowana Osteoglossum bichirrosum were noted recently, suggesting an additional WGD as a possible cause (Rozenfeld et al. 2019).

Transcriptome de novo assembly procedures include steps that can influence the observed number of duplicated genes, such as the clustering of the assembled contigs (Pasquier et al. 2016; Grabherr et al. 2011). Therefore, we collected all available reference genome assemblies matching the species of any of the transcriptome assemblies. The direct comparison of BUSCO estimates for de novo assemblies and reference genome-derived transcriptomes across 10 species revealed a high correspondence of the duplication level $\left(R^{2}=0.8\right.$ ) (supplementary fig. S3 and table S3, Supplementary Material online). In contrast, parameters reflecting technical differences of transcriptome generation show substantially lower correspondence, such as the number of fragmented $\left(R^{2}=0.02\right)$ or missing transcripts $\left(R^{2}=0.28\right)$. Clustering of BUSCO gene phylogenetic profiles across all considered species provides a more detailed picture (fig. 2). While a small group of 240 transcripts fails to assemble reliably (cluster I), likely reflecting specific sequence properties, the second group of 819 genes is detected as single-copy in the majority of species (cluster II). The largest cluster III, comprised of 2,096 genes, exhibits species-specific duplication patterns. Cluster IV contains 301 


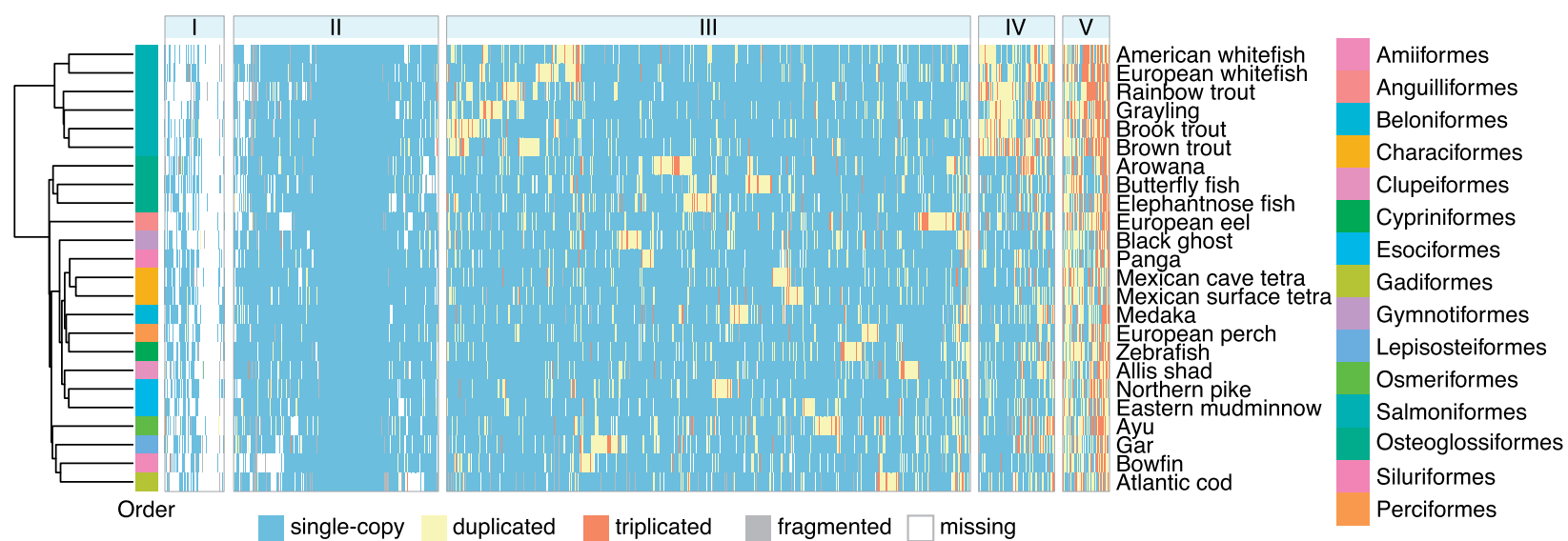

FIG. 2.-Phylogenetic profiling of universal single-copy orthologous genes in 24 de novo transcriptome assemblies. Genes are grouped into five clusters (top, $1-\mathrm{V}$ ) by profile similarity. The hierarchical clustering tree for all species is shown on the left together with the corresponding color-coded order.

genes duplicated mainly in salmonids and thus likely represent remnants of the salmonid-specific WGD (cluster IV). The remaining 184 genes appear almost consistently duplicated or triplicated across all considered species, again likely reflecting sequence properties (cluster $\mathrm{V}$ ). Both members of the Esociformes, the eastern mudminnow U. pygmaea, and the Northern pike $E$. lucius are placed next to each other in the hierarchical species clustering. Separating cluster III into subclusters reveals that both the eastern mudminnow and the Northern pike feature only small nonoverlapping groups of species-specific duplicated genes containing 47 and 76 genes, respectively (supplementary fig. S4 and table S4, Supplementary Material online).

\section{Comparative Genome Analyses of Esociformes Species Reveal Extensive Transposable Element Expansions}

We performed a direct comparative analysis of the genome composition of $U$. pygmaea using recently published shortread-based genome assemblies of five Esociformes species (Pan et al. 2021) and, in addition, the high-quality chromosome-scale genome assembly of $E$. lucius (supplementary table S5, Supplementary Material online). Due to the employed short-read sequencing and relatively low coverages, the assemblies for U. pygmaea, Esox masquinongy, Esox niger, Novumbra hubbsi, and Dallia pectoralis are fragmented and less complete (11-24\% BUSCO genes missing) compared to the chromosome-scale assembly of E. lucius (3\% missing). Nonetheless, the genome assembly sizes correspond well to direct measurements of genome size (fig. 3A). K-mer statistics obtained directly from the short-read data generally underestimate total genome size but yield the same relative pattern across species. In case of $E$. niger, no estimate could be obtained as the model fitting did not converge. In agreement with the transcriptome analysis, the assessment of BUSCO genes in all six genome assemblies shows low levels of duplication. We then characterized the repetitive sequence content of each genome aiming to test the hypothesis that transposable element activity might have caused a genome expansion exclusively in $U$. pygmaea. We, therefore, annotated repetitive sequences in all six genomes using a combined de novo repeat library (see Materials and Methods section). The observed repeat content in each genome scales with the total genome size (fig. 3B), with the highest repeat content of $64 \%$ in the largest genome of $U$. pygmaea and $37 \%$ of repeat content in the smallest genome of $N$. hubbsi (fig. 3C, stacked plot on the left). In general, DNA transposons make up the largest part of classified repeat sequences in all analyzed species, contributing about $19 \%$ of all genomic sequences in U. pygmaea, E. lucius, and E. masquinongy (supplementary table S4, Supplementary Material online). In contrast, E. niger, N. hubbsi, and D. pectoralis show lower DNA transposon abundances with $14.8 \%, 10.8 \%$, and $8 \%$, respectively. A similar pattern is observed for LINE elements, while SINE elements and LTRs are generally low in abundance. A principal component analysis of repeat abundances across all six species reveals that the repetitive sequence composition mirrors the phylogenetic relationships (supplementary fig. S5, Supplementary Material online), with high similarity between E. lucius and E. masquinongy, as well as between $N$. hubbsi and D. pectoralis. Both, U. pygmaea and E. niger, constitute outliers in repeat composition. Importantly, we observe a significant expansion of highly repetitive and species-specific sequences awaiting further characterization in $U$. pygmaea with $30.8 \%$ genomic abundance, compared to abundances in the range of $15.6-21.2 \%$ in all other species.

Similar to the repeat composition, repeat expansion histories also show significant differences between the considered species (fig. 3C). Here, the repeat expansion history is captured by the Kimura substitution level for each genomic copy of a repeat as a proxy for its age, since each copy accumulates mutations over time and diverges from its consensus sequence. The resulting divergence landscape suggests that the genome size increase of $U$. pygmaea results from an 
B

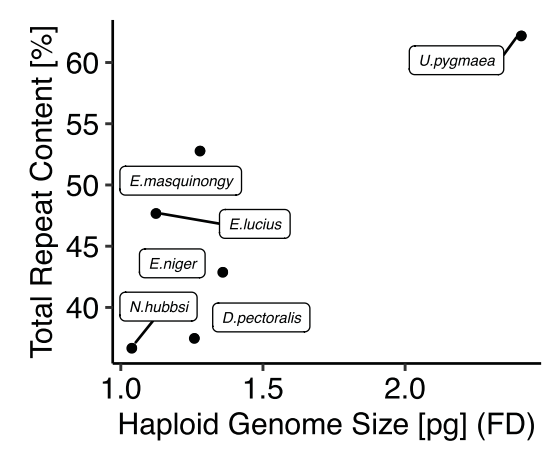

E. masquinongy

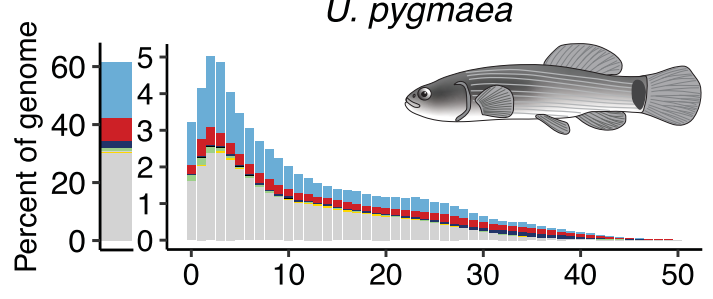

E. lucius
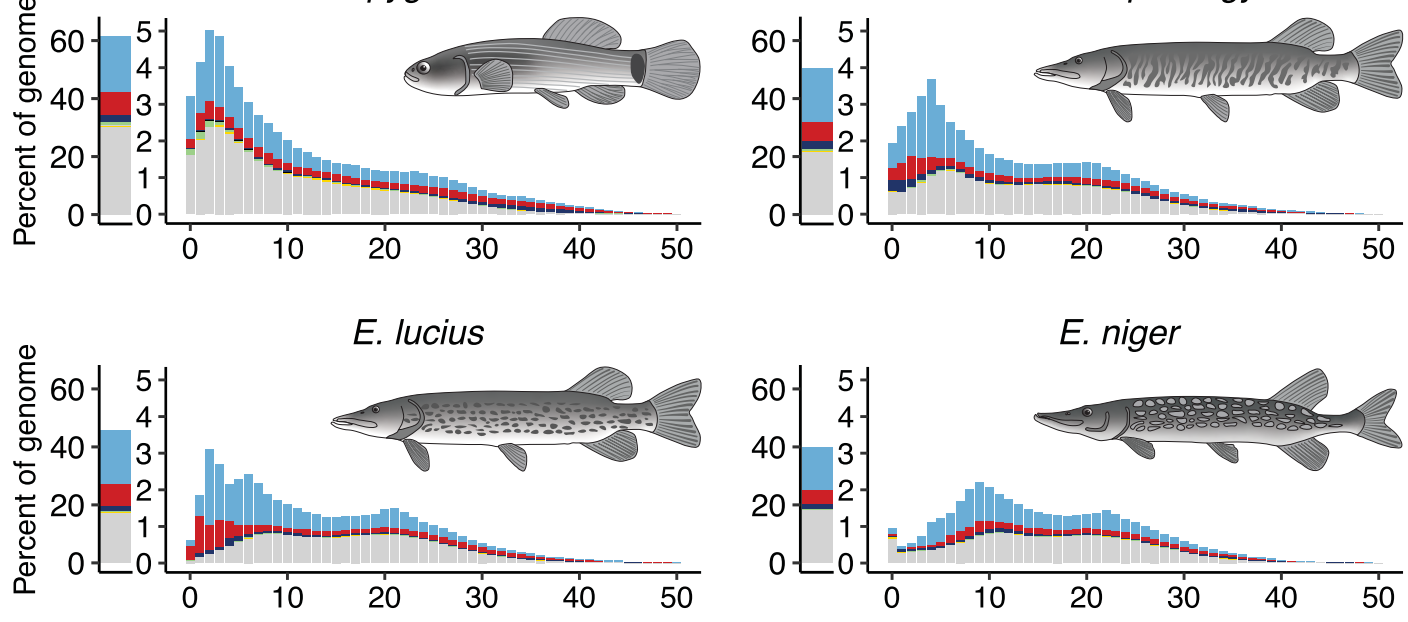

E. niger

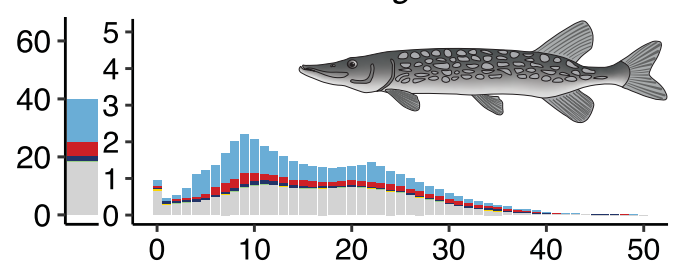

D. pectoralis
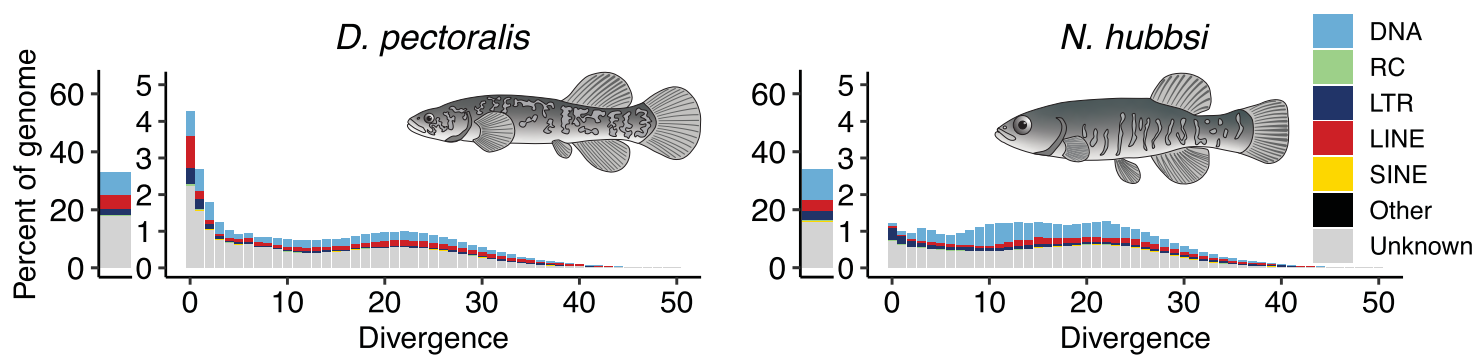

FIG. 3.-Genomic repeat sequence content and divergence landscapes in six Esociformes species. (A) Genome size measurements by Feulgen densitometry compared to genome assembly size (supplementary table S5, Supplementary Material online). (B) Genome assembly size compared to repetitive sequence content. (C) Total repetitive sequence content (left) and repeat copy divergence landscapes (right) as fraction of the total genome assembly for each of the six species, distinguishing the main repetitive sequence classes.

accumulation of unclassified repetitive sequence combined with the emergence of new DNA transposon copies, with $45 \%$ of the genomic repeat sequence showing a mean divergence below 8 (fig. 3C, top left). The most abundant repeat sequence in $U$. pygmaea is the Tc $1 /$ mariner element IC-Mifl (upygm-1-8) which makes up $1 \%(20.5 \mathrm{Mb})$ of the genome assembly with an average divergence of 8 (supplementary table S6, Supplementary Material online). The second most abundant repeat Tc1-2_Xt (upygm-1-14), with $14 \mathrm{Mb}$ and $0.7 \%$ of genomic sequence, is also a member of the Tc1/ mariner family and shows a very recent expansion pattern with an average divergence of 1.34. Esox masquinongy and E. lucius also show a notable recent expansion of DNA transposon sequences. Similar to U. pygmaea, IC-Mifl also contributes $1.3 \% / 11.7 \mathrm{Mb}$ to the total genome assembly of E. lucius, making it the third most abundant repeat only surpassed by Mariner-9 originally identified in S. salar and an RTE-1 nonlong-terminal-repeat element identified in E. lucius (supplementary table S6, Supplementary Material online). The prominence of IC-Mifl notwithstanding, the high total abundance 


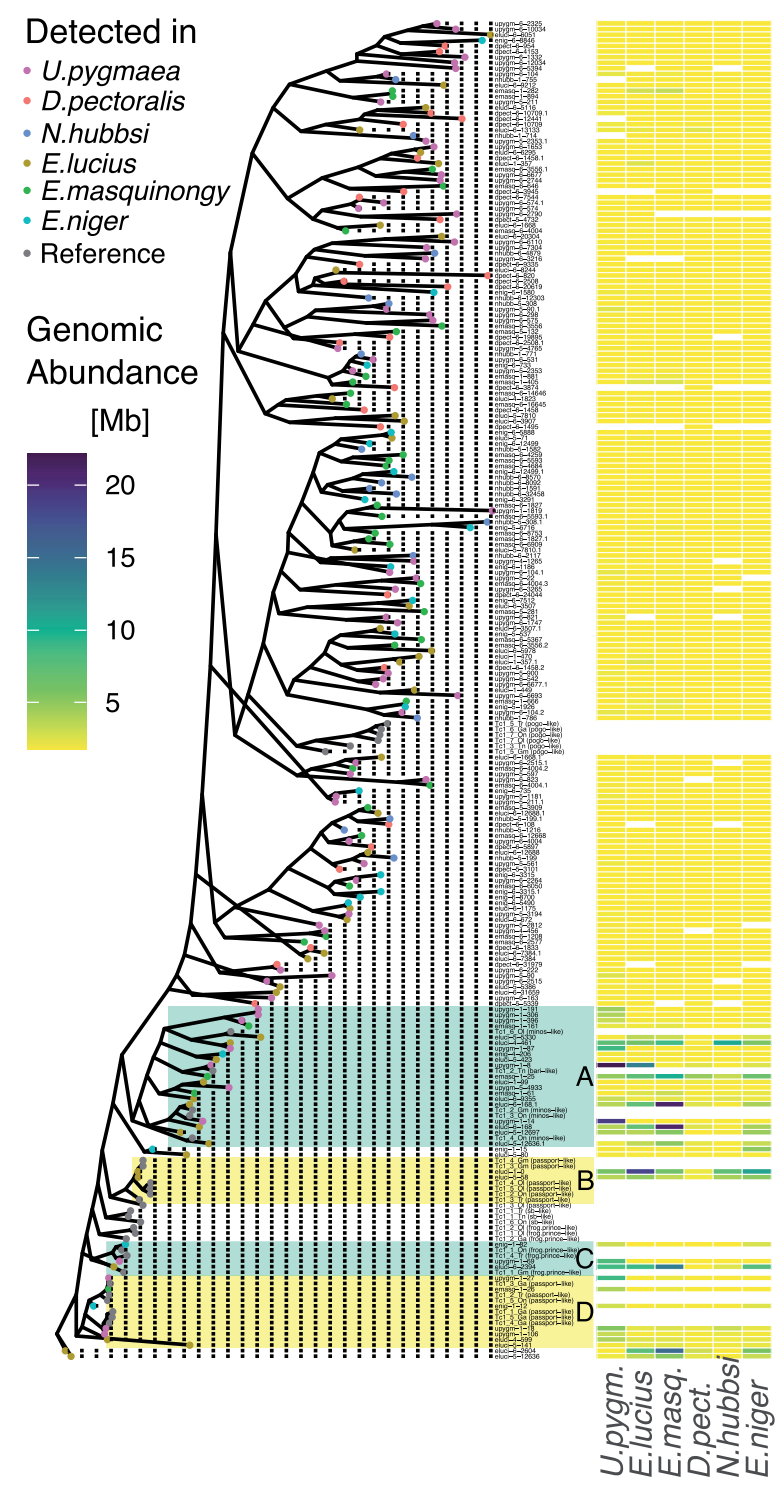

FIG. 4.-Phylogenetic relationship (left) and genomic abundance (right) of Tc1/mariner superfamily DNA transposons across six Esociformes species. The phylogenetic tree is based on transposase sequences from 206 repeats combined with 33 previously described (Gao et al. 2017) reference elements from six lineages (Pogo, Passport, SB, Frog Prince, Minos, and Bari). Clades containing most abundant elements are marked A-D. Genomic abundance values in Mb are based on full length repeat sequences, where elements not detected in the respective species are shown in white.

of DNA transposon sequence in the aforementioned species is caused by a multitude of active families. While the total repeat content of $D$. pectoralis is low, the divergence landscape reveals a currently ongoing repeat expansion with $27 \%$ of the total repeat content at divergences below 4 , which translates into $9.1 \%$ of the total genome sequence. In contrast to all other species, the expansion is caused by two LINE/RTEBovB elements with high abundance $(0.5$ and $0.2 \%$, respectively, of total genome) and low mean divergence (1.16 and 0.87) in combination with rRNA, tRNA, and unclassified sequences. This leaves $N$. hubbsi as the only species with a consistently low transposable element activity.

To gain a more detailed overview of the Tc1/mariner transposon superfamily activity, we constructed a phylogeny of all transposase sequences in our repeat library together with transposase sequences of a previously described Tc1/mariner reference set (Gao et al. 2017) in neoteleosts. This phylogeny was then complemented with the detected genomic abundances in the considered species (fig. 4). We find that the most highly abundant elements fall into the Minos-/Bari-like clade (fig. 4 clade A), such as the two most abundant elements of U. pygmaea (upygm-1-8, upygm-1-14), E. masquinongy (eluci-6-168), and N. hubbsi (eluci-4-461). Other more abundant lineages are passport (fig. 4 clade $B$ and D), with the most abundant element eluci-1-0 in $E$. lucius, and frog prince (fig. 4 clade C, upygm-1-69, eluci-6-2394).

Finally, we performed a manual curation of the 30 most abundant repeat sequences found in $U$. pygmaea, contributing a combined $101.87 \mathrm{Mb}$ to the genome assembly to test the extent to which the unclassified repeat sequence expansion can also be attributed to transposable element activity. We find TE-related structural features in 13 repeats, accounting for $48 \mathrm{Mb}$, in addition to a highly abundant tandem repeat and a satellite sequence (supplementary table S7, Supplementary Material online). With 11 out of 13 TErelated sequences, the vast majority show DNA transposon features. In general, we observe a consistent pattern of increased abundance across species in a small group of Tc1/ mariner elements mirroring the pattern of total genomic repeat abundance in the respective species.

\section{Fluorescence In Situ Hybridization}

Studied pike species mostly exhibit karyotypes composed of 50 monoarmed chromosomes ( $F N=50$ ). In turn, U. limi possesses only 22 metacentric and submetacentric chromosomes $(\mathrm{FN}=44)$, gradually decreasing in size. Fluorescence in situ hybridization (FISH) with PNA telomere probe revealed hybridization signals only at the very ends of all chromosomes in $E$. lucius and E. cisalpinus. In U. limi, apart from the terminal location of the FISH telomeric signals observed on all chromosomes, up to seven chromosomes exhibited interstitial telomeric sites (ITSS) in the pericentromeric locations. In four of these chromosomes, ITSs overlapped with DAPI positive regions. DAPI positive signals occurred on the q-arms below the pericentromeric regions and did not correspond with the ITSs for up to three other chromosomes (fig. 5A). The $5 S$ rDNA probe hybridized to three sites in a pericentromeric location on two metacentric chromosomes and centromeric location on one submetacentric chromosome in U. limi (fig. $5 B$ ). All 5S rDNA sites were DAPI-positive. 

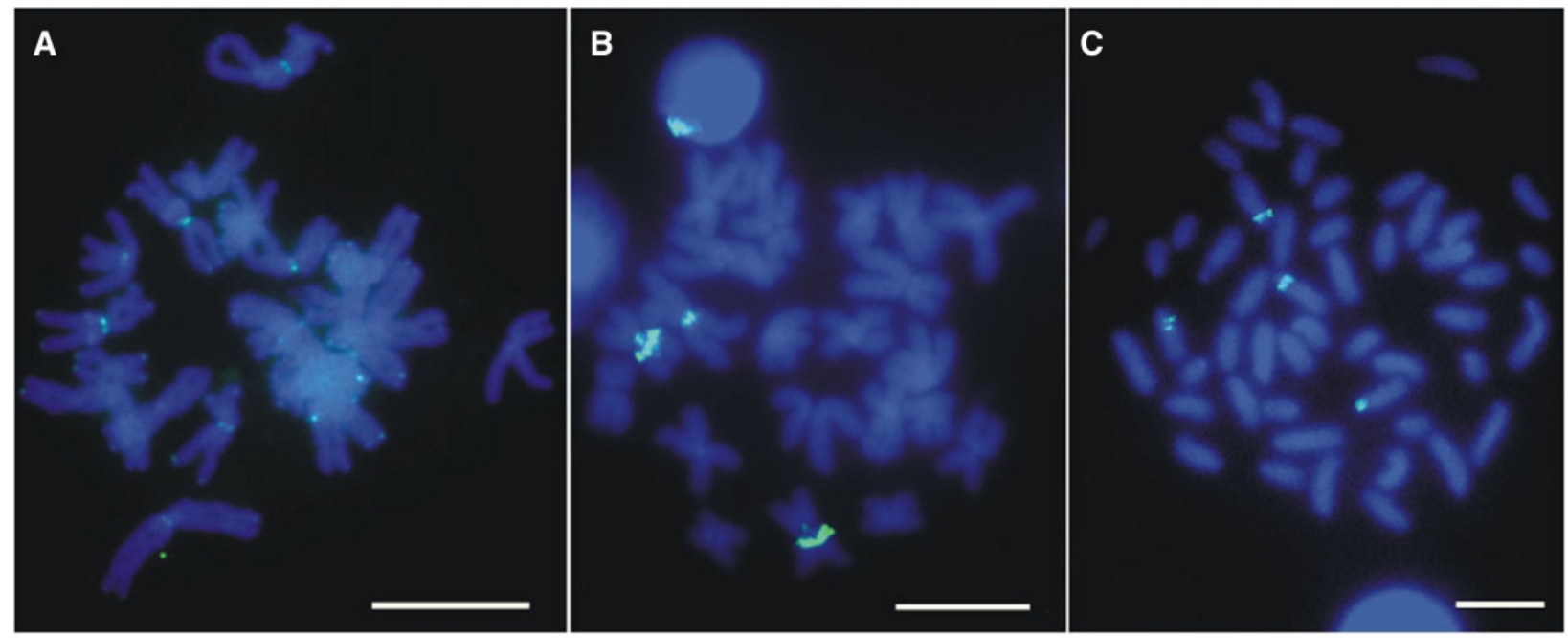

FIG. 5.-FISH with telomeric probes and $5 S$ rDNA. Chromosomes of Umbra limi after FISH with telomeric probe $(A)$ and $5 S$ rDNA probe $(B)$ and of Esox niger after FISH with $5 S$ rDNA probe (C). All chromosomes are counterstained with DAPI. The FISH with a telomeric probe in U. limi yielded stronger interstitial telomeric signals than the terminal ones. Bar $=10 \mu \mathrm{m}$.

\section{Molecular Analysis of 5S rDNA Genomic Fraction}

The slot blot quantification of $5 S$ rRNA genes indicates approximately 2,000 5S rDNA copies per Umbra genome with a slightly higher copy number in $U$. limi compared to $U$. krameri. Umbra thus has about ten times lower copy number of 5 S rRNA than both Esox species analyzed so far (fig. $6 A$ and $B$ ). These copies are arranged in tandems in both Umbra as well as in Esox indicated by ladders of bands after the digestion with Mspl restriction enzyme (not sensitive to CG methylation) (fig. 6C). Increased ladder length for both Umbra species reveals greater sequence divergence of Mspl (CCGG) sites compared to Esox. Digestion of DNAs with methylation-sensitive Hpall isoschizomere resulted in hybridization signals in high-molecular-weight regions with little to no ladders (fig. 6C), which is consistent with heavy methylation of internal Cs within the CCGG motifs.

\section{Discussion}

The sizes of eukaryotic genomes vary substantially with teleosts being no exception. The range of described cases of whole-genome duplication (WGD) events amongst teleosts makes this mechanism the main suspect when observing significant genome size increases. In contrast, we find that the genome expansion in the genus Umbra is not caused by a WGD but transposable element activity. At the same time, extensive chromosome fusion events accompanying genome expansion have occurred in some Umbra species. In the following, we discuss what sets these inconspicuous members of the order Esociformes apart from their sister lineage from an ecological and evolutionary point of view.

\section{Genome Expansion and Chromosome Fusions in Umbra sp}

Our results demonstrate that the European mudminnow species U. krameri underwent a genome expansion similar to the two North American Umbra sister species. This genome expansion is thus a common trait of the entire genus and can be localized in time prior to the split of the American and European Umbra lineages, setting the Umbridae family apart from their sister lineages Esox, Dallia, and Novumbra (Marić et al. 2017; Gregory 2020). The European species U. krameri retained the Umbra ancestral chromosome number $(2 n=$ $\mathrm{FN}=44$ ), which is even lower than the most common teleost ancestral $2 n \approx 50$ (Mank and Avise 2006; Sacerdot et al. 2018) chromosome number and might indicate an overall tendency towards chromosome number reduction in the genus Umbra. Paradoxically, the genome expansion in U. limi and $U$. pygmaea is accompanied by a further reduction in chromosome counts $(2 n=22)$ while maintaining the same number of chromosome arms ( $\mathrm{FN}=44)$. This reduction in the number of chromosomes without changes to the number of chromosome arms, as confirmed for $U$. limi, is consistent with Robertsonian fusion events. Accordingly, up to seven out of 22 biarmed chromosomes of $U$. limi exhibit interstitial telomeric sites (ITSS), which presumably are remnants of these fusions. Similar occurrences of telomeric DNA at nontelomeric sites as relics of chromosomal rearrangements including centric fusions (Robertsonian translocations), tandem fusions, and inversions (Ocalewicz 2013) have been observed at pericentromeric locations of fused mammalian and nonmammalian chromosomes (Ocalewicz et al. 2013; Meyne et al. 1990). Breaks in telomer-adjacent chromatin regions before the fusion event may explain the absence of telomeric repeat 


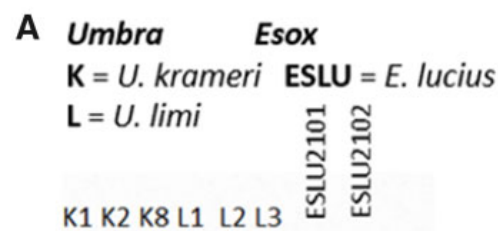
B $5 S$ rDNA genome proportion
(percentage of Esox)

K1

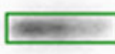

12
K2

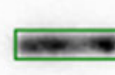

19

K8

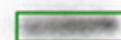

15

L1

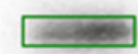

$\cdot 19$

L2

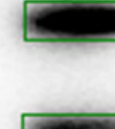

63

L3

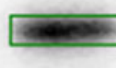

29

ESLU2101

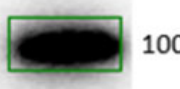

ESLU 2102

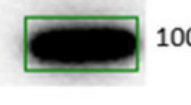

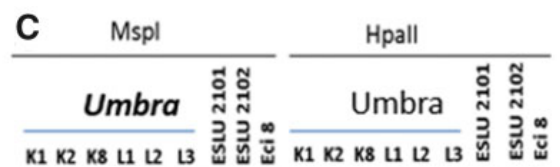

9

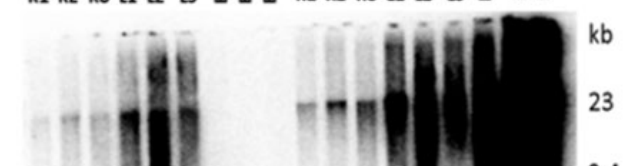

9.4

6.6

4.4

2.3

2.0

0.6

\section{Mbol restriction enzyme}

FIG. 6.-Genomic analysis of Umbra and Esox $5 S$ rDNA. (A) Southern blot hybridization. (B) Slot blot hybridization. Probe-5S rDNA insert from the $E$. cisalpinus clone a, K, U. krameri; L, U. limi; ESLU, E. lucius, (C) DNA methylation analysis of 5SrDNA in Umbra and Esox. Hpall is a methylation-sensitive isoschizomere of Mspl. Probe—5S rDNA insert from the E. cisalpinus (Eci) clone a. ESLU, Esox lucius; K, Umbra krameri; L, Umbra limi.

sequences at the remaining fusion sites (Nanda et al. 1995; Garagna et al. 1995). Furthermore, ITSs may undergo gradual loss and degeneration, leading to progressive shortening. So while FISH is currently the best approach to explore ITSs in situ, some interstitially located telomeric DNA repeats might be too short for detection (Slijepcevic 1998).

The low chromosome count in the North American Umbra species $(2 n=22)$ makes them part of a small group of fishes. Among about 32,000 fish species (Nelson et al. 2016), there are only thirteen fish species with $2 n<22$ chromosomes described to date, made up mainly of Cyprinodontiformes (six in Nothobranchiidae, one in Rivulidae) ranging between $2 n=16-20$. The lowest chromosome counts were described for the marine teleost species spark anglemouth (Sigmops bathyphilus, Stomiiformes, Gonostomatidae) (Post 1974) with $2 n=12$ and the freshwater teleost species chocolate gourami (Sphaerichthys osphromenoides, Osphronemidae, Anabantiformes) (Calton and Denton 1974; KorefSantibanez and Paepke 1994; Arai 2011) with $2 n=16$. Interestingly, the $S$. bathyphilus belongs to the order Stomiiformes, a member of the superorder Osmeromorpha that split after the earlier divergence of the superorder
Protacanthopterygii (i.e., Esociformes and Salmoniformes) (Nelson et al. 2016).

\section{Interplay between Cytogenomic and Ecological Traits}

The chromosome number of a species can be linked to its ecological traits. Specialized fishes were demonstrated to have smaller genomes than more generalized species within and across lineages (Hinegardner and Rosen 1972; Hardie and Hebert 2004). This constraint may be extended to the chromosome number and genome size in specialized species (Hardie and Hebert 2004), or constraints on genome size linked to the heightened developmental complexity of specialized species (Gregory 2002). Similar principles have been proposed for mammals (Qumsiyeh 1994) where species with higher $2 n$ or $\mathrm{FN}$ have a higher recombination rate because proper disjunction requires at least one chiasma per arm (Jensen-Seaman et al. 2004). Increased recombination rate not only leads to increased genetic variability, thereby allowing the utilization of a wider niche (Qumsiyeh 1994) but also to a genome size reduction (Nam and Ellegren 2012). On the other hand, decreased recombination rate favors the fixation 
of new mutations and thereby potential speciation (Qumsiyeh 1994). Furthermore, decreased recombination can cause the genome to expand due to the accumulation of transposon insertions (Dolgin and Charlesworth 2008). The interaction between transposon activity and recombination rate may create positive feedback, where the accumulation of transposon insertion sites contributes to further suppress recombination (Kent et al. 2017; Fedoroff 2012). The positive correlation between recombination rate and effective population size (Mugal et al. 2015) requires the consideration of the ecology of Umbra and Esox. Both genera's species belong to freshwater fishes at northern latitudes and hence are affected by bottlenecks and reduced effective population sizes within refugia during glaciation periods (Bernatchez and Wilson 1998). Low levels of genetic diversity in pikes have been described extensively (Skog et al. 2014) and may explain the extreme 5S rDNA amplification through genetic drift (Symonová et al. 2017). Comparably low estimates of the effective population size have been reported for U. krameri (Marić et al. 2017).

The ecology of mudminnows appears surprisingly similar. The American central mudminnow (U. limi) has been termed a "habitat specialist and resource generalist" (MartinBergmann and Gee 1985), referring to the densely vegetated and often overgrown and deoxygenated swampy habitats in which this species is found. Accessory air-breathing capabilities allow mudminnows to use such marginal habitats in which no, or only a few other fish species can be found. Otherwise, the central mudminnow uses a wide variety of small invertebrate prey. This brief ecological characterization can be extended to all other mudminnows (Kuehne and Olden 2014) which might be related to their low competitive abilities (Wanzenböck and Spindler 1995; Sehr and Keckeis 2017). In contrast, pikes are found in a wide variety of habitats, densely overgrown lentic waters to open waters in large lakes or even brackish waters and riverine habitats. Furthermore, they are rather specialized piscivorous during juvenile and adult life stages. Conversely, they might be called "habitat generalists and resource specialists." Whether such ecological differences may be related to genomic differences between mudminnows and pikes remain open.

Next to potentially ecological influences, there are also purely genomic factors driving genome size evolution. A common cause of genome size expansion is a WGD event (polyploidy) or repeat expansion due to transposon or satellite DNA. In the Umbra genus, however, the genome expansion was accompanied by a reduction in chromosome number, which suggests two scenarios

i. The Umbra lineage has experienced a rapid chromosome loss (disploidy) following an additional WGD. Evidence from plants suggests that repeated WGD cycles with subsequent chromosome loss may generate karyotypes with few chromosomes (Dodsworth et al. 2016). However, our comparative genome and transcriptome analyses do not support the existence of additional ohnologs in U. pygmaea. The observed number of duplicated protein-coding genes in the genome assemblies of $U$. pygmaea and its sister lineage $E$. lucius was similar, excluding WGD as cause of genome expansion in Umbra. While the completeness of the employed transcriptomes and genomes is relatively low, it is not plausible that specific ohnologs are disproportionately missing in both cases.

ii. Alternatively, the expansion of repeat sequences could explain the increased genome size. Evidence of such a repeat expansion was found in a sister lineage. The two species $E$. lucius and $E$. cisalpinus show an extreme amplification of $5 \mathrm{~S}$ rDNA across their chromosomes (Symonová et al. 2017) forming thus an rDNAome or an rDNA subgenome (Symonová 2019) while retaining their genome size and chromosome counts. This massive expansion of tandem repeats by several orders of magnitude must have happened over a short evolutionary time, as the related $E$. niger still features a low number of $5 \mathrm{~S}$ rDNA copies ancestral to the Esocidae lineage (Kovařík, unpublished data). While the Umbra lineage shows teleost-typical (Sochorová et al. 2018) $5 S$ rDNA site numbers on chromosomes, our comparative genome analysis of the $U$. pygmaea genome confirms a significantly elevated interspersed repeat content of $64 \%$, which is the result of a recent expansion of DNA transposon sequence and a range of unclassified repeat sequences. This peak is not only consistent with the pervasive DNA transposon expansion particularly in E. lucius, E. masquinongy, and $E$. niger. Manual curation of the top 30 highly abundant unclassified sequences reveals DNA transposon features, further supporting the observed DNA transposon expansion. There are numerous accounts that DNA transposons are the most abundant TE class amongst most fish genomes (Shao et al. 2019). Furthermore, Tc/mariner and hAT are the most common predominant TE superfamilies in fish genomes, next to L1, L2, and Gypsy class 1 superfamilies (Yuan et al. 2018). A comparison across the four model teleost species zebrafish, medaka, stickleback, and tetraodon demonstrated that the genome size difference is largely due to the differential expansion of Class II Tes, that is, DNA transposons (Gao et al. 2016). Recent expansions of DNA transposons have been observed in various fish species such as East African cichlids, where the youngest radiation to Lake Victoria, Pundamilia nyererei, is still experiencing an expansion of Tigger elements (Brawand et al. 2014). Amongst mammals, only bats experience active DNA transposon expansion (Ray et al. 2008).

but also with the generally high abundance of DNA transposons in most fish genomes (Shao et al. 2019). Manual curation of the top 30 highly abundant unclassified sequences reveals DNA transposon features, further supporting the observed DNA transposon expansion. The Alaska blackfish $D$. pectoralis constitutes an exception as 
it appears in the process of repeat expansion driven by LINE elements, rRNA, tRNA, and unclassified elements. Importantly, genome size variation is the net result of sequence gain and loss (Petrov et al. 2000; Symonová 2019; Kapusta et al. 2017) allowing for the possibility of sequence gain by TE expansion in U. pygmaea, or alternatively sequence loss of various extent in the other species. As the expansion peaks observed in all species except $N$. hubbsi appear to be relatively recent at divergences below ten percent, a sequence gain scenario is more plausible. As all but the E. lucius genome assembly are highly fragmented and lack the completeness of reference genome assemblies, it is however likely that the presented results underestimate the total repeat content, particularly for long repeat sequences. This fragmentation is also a likely explanation for the high cumulative abundance of unclassified repeat sequences in $U$. pygmaea, which taken together exceeds the contribution of DNA transposon sequence to the total assembly size. Furthermore, it is not possible to locate chromosomal fusion sites or potential duplications in the U. pygmaea assembly. Further analyses based on a platinum standard long read-based reference assemblies and an extensively curated library of repeat sequences are required to address these questions.

Here, we show that all members of the Umbra genus feature a significantly expanded genome, placing the expansion event prior to the separation between the European and North American species. However, only the North American species experienced Robertsonian chromosome fusion events, further reducing their chromosome number significantly. Taken together, our findings show that an activity peak mainly of Tc1/mariner DNA transposons significantly increased the size of the $U$. pygmaea genome. We furthermore find evidence of an ongoing repeat-driven genome expansion in the Alaska blackfish $D$. pectoralis. This makes the family Umbridae and the entire order Esociformes an attractive model system to study exclusively repeat-driven genome expansion and its potential role in speciation.

\section{Materials and Methods}

\section{Fish Sampling}

Ten individuals of Umbra krameri were descendants from individuals sampled between 1993-1997 from "Fadenbach" between Orth and Eckartsau (Danube Wetlands, National Park in Lower Austria) within a nature conservation project of the provincial government of Lower Austria (Wanzenböck and Spindler 1995). Ten individuals of U. limi and ten individuals of E. niger were sampled in Quebec, Canada, in autumn 2017. A local professional fisherman provided samples of two individuals of E. lucius at Lake Mondsee in 2016. Further specimens of $E$. lucius and $E$. cisalpinus were analyzed in our earlier study (Symonová et al. 2017). This included 28 eight-month- old specimens ( 12 males, nine females, and one unsexed) from the local fish farm (Olsztyn, Poland) sampled for cytogenetic analyses.

\section{Genome Size Determination by Flow Cytometry}

Ethanol fixed red blood cells (RBC) of $U$. krameri and E. lucius, respectively, were used for genome size determination following (Lamatsch et al. 2000) with chicken RBC as internal standard (C-value $1.25 \mathrm{pg}$ per nucleus). We determined genome size by flow cytometry using the Attune ${ }^{\mathrm{TM}}$ NxT Acoustic Focusing Cytometer (Thermo Fisher Scientific, Vienna, Austria). We applied instrument settings optimized using the AttuneR Cytometric Software. We used forward scatter (FSC) and VL1 violet fluorescence ( $405 \mathrm{~nm}$ excitation, bandpass filter $440 / 50 \mathrm{~nm}$ ) as triggers for all measurements (supplementary fig. S1, Supplementary Material online). We analyzed 20.000 cells or sample volumes of $0.1 \mathrm{ml}$ at a flow rate of $0.025 \mathrm{ml} / \mathrm{min}$, gating only singlet cells to minimize background. DAPI is an AT-specific stain, which can yield inaccurate results when the AT/GC content of the sample and internal standard differ significantly. Chicken and E. lucius RBC, however, show a very similar AT-content of $57.73 \%$ (Vinogradov 1998), and 56.8\%, (Borisova et al. 1974), respectively, suggesting that the underestimation of the total amount of DNA is negligible. Records on genome size come from the www.genomesize.com database (Gregory 2020) and the cited literature (supplementary table S1, Supplementary Material online).

\section{Comparative Transcriptome Analysis}

We analyzed 24 de novo transcriptome assemblies of the PhyloFish database (Pasquier et al. 2016): bowfin (Amia calva), gar (Lepisosteus oculatus), European eel (A. anguilla), butterflyfish (Pantodon buchholzi), arowana (Osteoglossum bicirrhosum), elephantnose fish (Gnathonemus petersi), Allis shad (Alosa alosa), zebrafish (Danio rerio), panga (Pangasianodon hypophthalmus), a black ghost (Apteronotus albifrons), cave Mexican tetra (Astyanax mexicanus), surface Mexican tetra (A. mexicanus), Northern pike (Esox lucius), Eastern mudminnow (U. pygmaea), grayling (Thymallus thymallus), European whitefish (Coregonus lavaretus), American (Lake) whitefish (Coregonus clupeaformis), brown trout (Salmo trutta), rainbow trout (Oncorhynchus mykiss), brook trout (Salvelinus fontinalis), Ayu (Plecoglossus altivelis), Atlantic cod (Gadus morhua), medaka (Oryzias latipes), and European perch (Perca fluviatilis). The completeness and duplication were assessed with BUSCO v4.1.4 (Simão et al. 2015) using the data set actinopterygii_odb10 containing 3640 genes (supplementary table S2, Supplementary Material online). For a subset of 10 species, reference genome assemblies and structural genome annotations are available at the NCBI reference sequence database (supplementary table S3, Supplementary Material online). For this subset, de novo 
transcriptome assembly completeness and duplication were compared to reference-genome-derived transcriptomes using identical BUSCO settings. Gene counts were separately compared for each of the five gene categories (complete, complete-single-copy, complete-duplicated, fragmented, missing) using the coefficient of determination (supplementary fig. S3, Supplementary Material online). A phylogenetic profile matrix was assembled representing the number of orthologous copies detected for each of the 3640 BUSCO genes across 24 transcriptome assemblies. Hierarchical clustering of the phylogenetic profile matrix was performed using Manhattan distance and the "ward.D2" method implemented in R v3.6.1 to obtain five main gene clusters and 26 subclusters of main cluster III.

\section{Comparative Genome Analysis}

The five published (Pan et al. 2021) short-read based scaffoldlevel genome assemblies of the Esocidae species $U$. pygmaea, $D$. pectoralis, N. hubbsi, E. niger, and E. masquinongy, as well as a chromosome-scale reference genome assembly of $E$. lucius, were used for a comparative genome analysis (see supplementary table S5, Supplementary Material online for accession numbers). The haploid genome size for all shortread data sets was estimated using jellyfish v2.3 (Marçais and Kingsford 2011) to generate the $k$-mer histograms $(k=21)$ and then fitting a mixture model with GenomeScope v1.0 (Vurture et al. 2017). For each of the six genomes, a de novo repeat library was generated using RepeatModeler 2.0.1 (Smit and Hubley 2008), which yielded between 2,547 and 4,983 sequences per species. These libraries were then combined and clustered using usearch v11.0.667 with $80 \%$ similarity cutoff to obtain a single nonredundant repeat library for the order Esociformes, which yielded a total of 14,856 sequences. Censor 4.2.29 (Jurka et al. 1996) was used to identify de novo sequences in addition to the RepeatModeler classification and annotation with RepeatMasker and RepBase 26.01. This library was then used as a reference to annotate repeat sequences with RepeatMasker 4.1.1 (Smit et al. 2010). Repeats containing known TE coding domains were identified by predicting open reading frames using Emboss v6.6.0.0 and comparing the resulting sequences against the transposase protein sequence collection provided with LTR_retriever (Ou and Jiang 2018) via blast. Requiring hits longer than 150 amino acids and with e-value $<1 \mathrm{e}^{-10}$ yielded 116 hits in LINE elements and 206 hits in sequences not classified as LINE elements, the latter of which were retained for further analysis. This procedure was also used to extract 33 transposase sequences of six previously described neoteleost Tc1/mariner lineages (Gao et al. 2017). All 239 sequences were then aligned with Clustal Omega v1.2.4 (Sievers and Higgins 2018), and a maximum likelihood phylogenetic tree was calculated with RAxML 8.2.12 (Stamatakis 2006) using a variable time amino acid substitution model with gamma-distributed rate heterogeneity. Analysis and visualization of all data were performed using $R$ 3.6.1. The 30 most abundant $U$. pygmaea repeat sequences of the de novo library which did not show significant similarity with known TEs were selected for further manual curation. Firstly, we tested if the unclassified sequences include local context sequences in addition to the core repetitive tract. To that end, the repetitive sequence was used as a query in a Blastn (Altschul et al. 1997) search against the U. pygmaea genome assembly. Two or more matching loci located on different contigs/scaffolds were extracted including $10 \mathrm{Kbp}$ up- and downstream of the matching sequence. These hit sequences were then compared to each other in a dot plot analysis using the dotter (Sonnhammer and Durbin 1995), allowing the exact definition of the entire repetitive element. Complete elements were then analyzed for similarity with known TEs as defined in the latest version of RepBase (Bao et al. 2015) and/or for the presence of structural features typical of known TE elements such as the inverted repeats of DNA TEs. This allowed the association of 13 elements to a TE class including 2 cases with only diagnostic structural features (supplementary table S7, Supplementary Material online). In addition, one satellite and one tandem repeat sequence were identified, leaving a total of 14 sequences unclassified. The curated sequences are provided at Zenodo (DOI 10.5281/zenodo.5166944) and https://github.com/roblehmann/umbridae_genome_expansion.

\section{Chromosome Analyses and Fluorescence in Situ Hybridization}

Telomeric DNA repeats on chromosomes of Umbra and Esox species were detected by FISH, using a telomere PNA (peptide nucleic acid) FISH Kit/FITC (DAKO, Denmark) according to the manufacturer's protocol. Slides with the metaphase spreads were washed with buffer (Tris-buffered saline, pH 7.5) for 2 min, immersed in 3.7\% formaldehyde in 1x TBS for $2 \mathrm{~min}$, washed twice in TBS for $5 \mathrm{~min}$ each, and treated with the Pretreatment solution including Proteinase $\mathrm{K}$ (DAKO) for $10 \mathrm{~min}$. Afterward, slides were washed twice in TBS buffer for 5 min each, dehydrated through a cold $(-20$ ${ }^{\circ} \mathrm{C}$ ) ethanol series $(70 \%, 85 \%, 96 \%)$ for $1 \mathrm{~min}$, and air-dried at room temperature. Ten microliters of FITC PNA telomere probe mix (DAKO) was dropped on the prepared slides and covered with the coverslip. Chromosomal DNA was denatured at $85^{\circ} \mathrm{C}$ for 5 min under the coverslip in the presence of the PNA probe. The hybridization reaction took place in the darkness at room temperature for $90 \mathrm{~min}$. After hybridization, the coverslips were gently removed by immersion of slides in the Rinse Solution (DAKO) for $1 \mathrm{~min}$. Slides were then washed in the Wash Solution (DAKO) for $5 \mathrm{~min}$ at $65^{\circ} \mathrm{C}$ and dehydrated by immersion through a series of cold ethanol washes of $70 \%, 85 \%, 96 \%$ for $1 \mathrm{~min}$ each and air-dried at room temperature. For the counterstaining, chromosomes were 
mounted in the VECTASHIELD Antifade Mounting Medium containing DAPI (Vector Laboratories, USA).

Metaphase plates were analyzed under a Zeiss Axio Imager A1 microscope equipped with a fluorescent lamp and a digital camera. The images were electronically processed using the Band View/FISH View software (Applied Spectral Imaging, Galiliee, Israel). FISH with $5 S$ rDNA probe was performed according to Fujiwara et al. (1998) with slight modification (Kirtiklis et al. 2014). A 5 S rDNA probe was obtained via PCR with forward primer 5S-1: 5' -TACGCC CGATCT CGT CCG ATC $-3^{\prime}$ and reverse primer 5S-2: 5'-CAG GCTGGT ATG GCC GTA AGC-3' (Pendas et al. 1994). The PCR reaction was carried out in a $50 \mu \mathrm{l}$ reaction volume containing 1.25 U GoTaq Flexi DNA Polymerase (Promega, USA), $10 \mu$ l of 5X Flexi Reaction Buffer (Promega, USA), $100 \mu \mathrm{M}$ of each dNTP (Promega, USA), $3 \mathrm{mM}$ of $\mathrm{MgCl}_{2}$ (Promega, USA), $10 \mathrm{pM}$ of each primer, $2 \mu$ l of DNA template, and nuclease-free water. PCR product, obtained after 30 cycles of amplification and annealing at $55^{\circ} \mathrm{C}$, was purified using the GeneElute PCR Clean-Up Kit (Sigma-Aldrich, USA), then labeled with biotin16-dUTP (Roche, Germany) by nick-translation method (Roche, Switzerland). In situ hybridization with $150 \mathrm{ng}$ of rDNA probe per slide was performed with RNase-pretreated and formamide-denaturated chromosome slides. A posthybridization wash was performed at $37^{\circ} \mathrm{C}$ for $20 \mathrm{~min}$. Chromosome slides were subjected to the detection with avidin-FITC (Roche, Basel, Switzerland) and then counterstained with DAPI in VECTASHIELD Antifade Mounting Medium (Vector Laboratories, USA). At least 15 metaphase chromosome spreads from each specimen were analyzed using a Nikon Eclipse 90i (Nikon, Japan) microscope equipped with epi-fluorescence. Pictures were acquired using a monochromatic ProgRes MFcool camera (Jenoptic, Germany) controlled by a Lucia software ver. 2.0 (Laboratory Imaging, Czech Republic). Postprocessing elaboration of all the pictures was made based on CorelDRAW Graphics Suite 11 (Corel Corporation, Canada).

\section{Southern and Slot Blot Hybridizations of 5 rDNA}

The procedure followed the protocol described by Koukalova et al. (2010). The $5 S$ rDNA probe was a 243 bp insert of the 5S_Eci_a clone (GenBank KX965716) from E. cisalpinus. The plasmid insert was amplified and labeled with the 32P-dCTP (DekaPrime kit, Fermentas, Lithuania). The probe was hybridized at high stringency conditions (washing $2 \times$ SSC, $0.1 \%$ SDS followed by $0.1 \times$ SSC, $0.1 \%$ SDS at $65^{\circ} \mathrm{C}$ ). The hybridization signals were visualized by Phosphor imaging (Typhoon 9410, GE Healthcare, PA, USA), and signals were quantified using ImageQuant software (GE Healthcare, PA, USA). The copy number of $5 S$ rDNA genes was estimated using slot blot hybridization. Briefly, the DNA concentration was estimated spectrophotometrically at OD260nm using a Nanodrop 3300 fluorospectrometer (Thermo Fisher Scientific, USA).
Concentrations were verified by the electrophoresis in agarose gels using dilutions of lambda DNA as standards. The three dilutions of genomic DNA $(100,50$, and $25 \mathrm{ng})$, together with serial dilutions of unlabeled plasmid inserts corresponding to the $5 S$ monomers (GenBank KX965715-6). Each aliquot was denatured in $0.4 \mathrm{M} \mathrm{NaOH}$ and blotted onto a positively charged Nylon membrane (Hybond XC, GE Healthcare, USA) using a vacuum slot blotter (SchleicherSchuell, Germany). The probe and the hybridization conditions and visualization of signals were the same as described above.

\section{DNA Methylation Analysis of 55 rDNA}

Purified genomic DNA samples of U. krameri (K1-K3), U. limi (L1-L3), and from $E$. lucius (two individuals as a control) were digested with methylation-sensitive Hpall (sensitive to CG methylation) and its methylation-insensitive Mspl isoschizomere (both enzymes are cutting at CCGG). The restriction fragments were hybridized on blots with the alpha[P $\left.{ }^{32}\right]$ dCTP-labelled $5 S$ rDNA probe. Control of digestion efficiency was carried out by spiking the Esox genomic DNA with a nonmethylated plasmid DNA (pBluescript, Stratagen) and subsequent hybridization with a plasmid probe. Both Mspl and Hpall enzymes yielded expected restriction fragments (not shown).

\section{Supplementary Material}

Supplementary data are available at Genome Biology and Evolution online.

\section{Acknowledgments}

This study was supported by the Tyrolean funds project with contract Nr. UNI-0404/2015 to R.S. The authors are also grateful to the "Excelence projekt PřF UHK 2209/2018" and the Czech Science Foundation (19-03442S) for financial support. R.S. acknowledges the EuroTech Postdoc Programme which is co-funded by the European Commission under its framework programme Horizon 2020, Grant Agreement number 754462. We acknowledge Petr Ráb for discussion on genome size in the Umbra genus, Guillaume Côté for fishing Umbra and Esox in Québec, and we also acknowledge Maria Pichler of UIBK for her technical support.

\section{Author Contributions}

R.S. conceptualized and supervised the study and provided the cytogenomic context; R.S., A.K., R.L., and D.K.L. methodized the study and wrote the original draft; R.L. analyzed fish transcriptomes and genomes; R.L. and A.Z. analyzed transposable elements; A.K. performed southern blot and slot blot hybridizations; D.K.L. performed the flow cytometry genome size determination; J.W. provided the ecological and 
phylogenetic context of the study and specimens for analyses; L.B. organized sampling, provided specimens for analyses, and reviewed the manuscript; K.O. and L.K. performed the cytogenetic analyses; R.L., R.S., D.K.L., K.O., L.K., J.W., and J.T. wrote, reviewed, and edited the study; R.S. acquired funding for the study.

\section{Data Availability}

The de novo repeat library and manually curated sequences are available via https://github.com/roblehmann/umbridae_ genome_expansion and Zenodo (DOI 10.5281/ zenodo.5166944).

\section{Literature Cited}

Altschul SF, et al. 1997. Gapped BLAST and PSI-BLAST: a new generation of protein database search programs. Nucleic Acids Res. 25(17):3389-3402.

Arai R. 2011. Fish karyotypes: a check list. Japan: Springer.

Bao W, Kojima KK, Kohany O. 2015. Repbase update, a database of repetitive elements in eukaryotic genomes. Mob DNA. 6:11.

Bernatchez L, Wilson CC. 1998. Comparative phylogeography of Nearctic and Palearctic fishes. Mol Ecol. 7(4):431-452.

Borisova OF, Razjivin AP, Zaregorodzev VI. 1974. Evidence for the quinacrine fluorescence on three at pairs of DNA. FEBS Lett. 46(1):239-242.

Brawand D, et al. 2014. The genomic substrate for adaptive radiation in African cichlid fish. Nature 513(7518):375-381.

Calton MS, Denton TE. 1974. Chromosomes of the chocolate gourami: a cytogenetic anomaly. Science 185(4151):618-619.

Dodsworth S, Chase MW, Leitch AR. 2016. Is post-polyploidization diploidization the key to the evolutionary success of angiosperms? Bot J Linn Soc. 180(1): 1-5.

Dolgin ES, Charlesworth B. 2008. The effects of recombination rate on the distribution and abundance of transposable elements. Genetics 178(4):2169-2177

Ellegren H. 2004. Microsatellites: simple sequences with complex evolution. Nat Rev Genet. 5(6):435-445.

Elliott TA, Gregory TR. 2015. Do larger genomes contain more diverse transposable elements? BMC Evol Biol. 15:1-10.

Fedoroff NV. 2012. Transposable elements, epigenetics, and genome evolution. Science 338(6108):758-767.

Fontdevila A. 2011. The dynamic genome: a Darwinian approach. Oxford: OUP.

Fujiwara A, Abe S, Yamaha E, Yamazaki F, Yoshida MC. 1998. Chromosomal localization and heterochromatin association of ribosomal RNA gene loci and silver-stained nucleolar organizer regions in salmonid fishes. Chromosom Res. 6(6):463-471.

Gao B, et al. 2017. Characterization of autonomous families of Tc1/mariner transposons in neoteleost genomes. Mar Genomics. 34:67-77.

Gao B, et al. 2016. The contribution of transposable elements to size variations between four teleost genomes. Mob DNA. 7:1-16.

Garagna S, et al. 1995. Robertsonian metacentrics of the house mouse lose telomeric sequences but retain some minor satellite DNA in the pericentromeric area. Chromosoma 103(10):685-692.

Gilbert C, Williams J. 2002. Field guide to fishes. North America. New York: Alfred A. Knopf.

Grabherr MG, et al. 2011. Full-length transcriptome assembly from RNASeq data without a reference genome. Nat Biotechnol. 29(7):644-652.

Gregory TR. 2020. Animal genome size database. Available from: http:// www.genomesize.com.
Gregory TR. 2002. Genome size and developmental complexity. Genetica 115(1):131-146.

Gregory TR, Hebert PDN. 1999. The modulation of DNA content: proximate causes and ultimate consequences. Genome Res. 9(4):317-324.

Hardie DC, Hebert PDN. 2004. Genome-size evolution in fishes. Can J Fish Aquat Sci. 61(9):1636-1646.

Hinegardner R. 1968. Evolution of cellular DNA content in teleost fishes. Am Nat. 102(928):517-523.

Hinegardner R, Rosen DE. 1972. Cellular DNA content and the evolution of teleostean fishes. Am Nat. 106(951):621-644.

Jensen-Seaman $\mathrm{Ml}$, et al. 2004. Comparative recombination rates in the rat, mouse, and human genomes. Genome Res. 14(4):528-538.

Jurka J, Klonowski P, Dagman V, Pelton P. 1996. CENSOR - a program for identification and elimination of repetitive elements from DNA sequences. Comput Chem. 20(1):119-121.

Kapusta A, Suh A, Feschotte C. 2017. Dynamics of genome size evolution in birds and mammals. Proc Natl Acad Sci U S A. 114(8):E1460-E1469.

Kent TV, Uzunović J, Wright SI. 2017. Coevolution between transposable elements and recombination. Philos. Trans. R. Soc. B Biol. Sci. 372:1736. doi: 10.1098/rstb.2016.0458.

Khoshkholgh M, Alireza A, Sajad N. 2015. Karyotypic characterization of the pike, Esox lucius from the south Caspian Sea basin. Iran J Anim Biosyst. 11:43-49.

Kirtiklis L, Ocalewicz K, Wiechowska M, Boroń A, Hliwa P. 2014. Molecular cytogenetic study of the European bitterling Rhodeus amarus (Teleostei: cyprinidae: acheilognathinae). Genetica 142(2): 141-148.

Koref-Santibanez S, Paepke H. 1994. Karyotypes of the Trichogasterinae Liem (Teleostei, Anabantoidei.). In: Abstr. VIII Congr. Soc. Eur. Ichthyol. Oviedo. p. 55.

Koukalova B, et al. 2010. Fall and rise of satellite repeats in allopolyploids of Nicotiana over C. 5 million years. New Phytol. 186(1):148-160.

Kuehne LM, Olden JD. 2014. Ecology and conservation of Mudminnow species worldwide. Fisheries 39(8):341-351.

Lamatsch DK, Steinlein C, Schmid M, Schartl M. 2000. Noninvasive determination of genome size and ploidy level in fishes by flow cytometry: detection of triploid Poecilia formosa. Cytometry 39(2):91-95.

Li JT, et al. 2015. The fate of recent duplicated genes following a fourthround whole genome duplication in a tetraploid fish, common carp (Cyprinus carpio). Sci Rep. 5:1-9.

Liedtke HC, Gower DJ, Wilkinson M, Gomez-Mestre I. 2018. Macroevolutionary shift in the size of amphibian genomes and the role of life history and climate. Nat Ecol Evol. 2(11):1792-1799.

Lien S, et al. 2016. The Atlantic salmon genome provides insights into rediploidization. Nature 533(7602):200-205.

Lu J, Peatman E, Tang H, Lewis J, Liu Z. 2012. Profiling of gene duplication patterns of sequenced teleost genomes: evidence for rapid lineagespecific genome expansion mediated by recent tandem duplications. BMC Genomics. 13:246-210.

Lynch M. 2007. The origins of genome architecture. Oxford: Oxford University Press (OUP).

Macqueen DJ, Johnston IA. 2014. A well-constrained estimate for the timing of the salmonid whole genome duplication reveals major decoupling from species diversification. Proc Biol Sci. 281(1778):20132881.

Mank JE, Avise JC. 2006. Phylogenetic conservation of chromosome numbers in Actinopterygiian fishes. Genetica 127(1-3):321-327.

Marburger S, et al. 2018. Whole genome duplication and transposable element proliferation drive genome expansion in Corydoradinae catfishes. Proc R Soc B. 285(1872):20172732.

Marić S, et al. 2017. Phylogeography and population genetics of the European mudminnow (Umbra krameri) with a time-calibrated phylogeny for the family Umbridae. Hydrobiologia 792(1):151-168. 
Martin-Bergmann KA, Gee JH. 1985. The central mudminnow, Umbra limi (Kirtland), a habitat specialist and resource generalist. Can J Zool. 63(8):1753-1764.

Meyne J, et al. 1990. Distribution of non-telomeric sites of the (TTAGGG)n telomeric sequence in vertebrate chromosomes. Chromosoma 99(1):3-10.

Mugal CF, Weber CC, Ellegren H. 2015. GC-biased gene conversion links the recombination landscape and demography to genomic base composition. Bioessays 37(12):1317-1326.

Nam K, Ellegren H. 2012. Recombination drives vertebrate genome contraction. PLoS Genet. 8(5):e1002680.

Nanda I, Schneider-Rasp S, Winking H, Schmid M. 1995. Loss of telomeric sites in the chromosomes of Mus musculus domesticus (Rodentia: muridae) during Robertsonian rearrangements. Chromosome Res. 3(7):399-409.

Nelson JS, Grande TC, Wilson MVH. 2016. Fishes of the world, 5th ed. Hoboken: Wiley.

Ocalewicz K, et al. 2013. Pericentromeric location of the telomeric DNA sequences on the European grayling chromosomes. Genetica 141(10-12):409-416.

Ocalewicz K. 2013. Telomeres in fishes. Cytogenet Genome Res. 141(23):114-125.

Ohno S. 2013. Evolution by gene duplication. Berlin Heidelberg: Springer.

Ou S, Jiang N. 2018. LTR_retriever: a highly accurate and sensitive program for identification of long terminal repeat retrotransposons. Plant Physiol. 176(2):1410-1422.

Pan Q, et al. 2021. The rise and fall of the ancient northern pike master sex determining gene. Elife 10:1-50.

Pasquier J, et al. 2016. Gene evolution and gene expression after whole genome duplication in fish: the PhyloFish database. BMC Genomics. 17:368.

Van de Peer Y, Mizrachi E, Marchal K. 2017. The evolutionary significance of polyploidy. Nat Rev Genet. 18(7):411-424.

Pendas AM, Moran P, Freije JP, Garcia-Vazquez E. 1994. Chromosomal mapping and nucleotide sequence of two tandem repeats of Atlantic salmon 5S rDNA. Cytogenet Cell Genet. 67(1):31-36.

Petrov DA, Sangster TA, Johnston JS, Hartl DL, Shaw KL. 2000. Evidence for DNA loss as a determinant of genome size. Science 287(5455):1060-1062.

Post A. 1974. Ergebnisse der Forschungsreisen des FFS "Walther Herwig" nach Südamerika. XXXIV. Die Chromosomen von drei Arten aus der Familie Gonostomatidae (Osteichthyes, Stomiatoidei). Arch Fischwiss. 25:51-55.

Pritham EJ. 2009. Transposable elements and factors influencing their success in eukaryotes. J Hered. 100(5):648-655.

Qumsiyeh MB. 1994. Evolution of number and morphology of mammalian chromosomes. J Hered. 85(6):455-465.

Ráb P. 2004. Karyotype evolution in fishes of the order Esociformes [Habilitation Thesis]. Prague: Charles University in Prague (in Czech).

Ray D, et al. 2008. Multiple waves of recent DNA transposon activity in the bat, Myotis lucifugus. Genome Res. 18(5):717-728.

Rondeau EB, et al. 2014. The genome and linkage map of the Northern Pike (Esox lucius): conserved synteny revealed between the salmonid sister group and the Neoteleostei. PLoS One. 9(7):e102089.

Rozenfeld C, et al. 2019. De novo European eel transcriptome provides insights into the evolutionary history of duplicated genes in teleost lineages. PLoS One. 14(6):e0218085.
Sacerdot C, Louis A, Bon C, Berthelot C, Roest Crollius H. 2018. Chromosome evolution at the origin of the ancestral vertebrate genome. Genome Biol. 19(1):166.

Sehr M, Keckeis H. 2017. Habitat use of the European mudminnow Umbra krameri and association with other fish species in a disconnected Danube side arm. J Fish Biol. 91(4):1072-1093.

Shao F, Han M, Peng Z. 2019. Evolution and diversity of transposable elements in fish genomes. Sci. Rep. 9:1-8.

Sievers F, Higgins DG. 2018. Clustal Omega for making accurate alignments of many protein sequences. Protein Sci. 27(1):135-145.

Simão FA, Waterhouse RM, loannidis P, Kriventseva EV, Zdobnov EM. 2015. BUSCO: assessing genome assembly and annotation completeness with single-copy orthologs. Bioinformatics 31(19):3210-3212.

Skog A, Vøllestad LA, Stenseth NC, Kasumyan A, Jakobsen KS. 2014. Circumpolar phylogeography of the northern pike (Esox lucius) and its relationship to the Amur pike (E. reichertii). Front Zool. 11:67.

Slijepcevic P. 1998. Telomeres and mechanisms of Robertsonian fusion. Chromosoma 107(2):136-140.

Smit AFA, Hubley R. 2008. RepeatModeler Open-1.0. Available from: www.repeatmasker.org.

Smit AFA, Hubley R, Green P. 2010. RepeatMasker Open-4.0. Available from:www.repeatmasker.org.

Sochorová J, Garcia S, Gálvez F, Symonová R, Kovařík A. 2018. Evolutionary trends in animal ribosomal DNA loci: introduction to a new online database. Chromosoma 127(1):141-150.

Sonnhammer ELL, Durbin R. 1995. A dot-matrix program with dynamic threshold control suited for genomic DNA and protein sequence analysis. Gene 167(1-2):GC1.

Stamatakis A. 2006. RAxML-VI-HPC: maximum likelihood-based phylogenetic analyses with thousands of taxa and mixed models. Bioinformatics 22(21):2688-2690.

Sun C, et al. 2012. LTR retrotransposons contribute to genomic gigantism in plethodontid salamanders. Genome Biol Evol. 4(2):168-183.

Symonová R, et al. 2017. Higher-order organisation of extremely amplified, potentially functional and massively methylated $5 S$ rDNA in European pikes (Esox sp.). BMC Genomics. 18(1):391.

Symonová R. 2019. Integrative rDNAomics-importance of the oldest repetitive fraction of the eukaryote genome. Genes (Basel). 10(5):345.

Tenaillon MI, Hollister JD, Gaut BS. 2010. A triptych of the evolution of plant transposable elements. Trends Plant Sci. 15(8):471-478.

Vinogradov AE. 1998. Genome size and GC-percent in vertebrates as determined by flow cytometry: the triangular relationship. Cytometry 31(2):100-109.

Vurture GW, et al.. 2017. GenomeScope: fast reference-free genome profiling from short reads. Bioinformatics. 33(14):2202-2204.

Wanzenböck J, Spindler T. 1995. Rediscovery of Umbra krameri (Walbaum, 1972) in Austria and subsequent investigations. Ann. des Naturhistorischen Museums Wien. Ser. B Für Bot. Zool. 97:450-457.

Wong WY, et al. 2019. Expansion of a single transposable element family is associated with genome-size increase and radiation in the genus Hydra. Proc Natl Acad Sci U S A. 116(46):22915-22917.

Yuan Z, et al. 2018. Comparative genome analysis of 52 fish species suggests differential associations of repetitive elements with their living aquatic environments. BMC Genomics 19(1):1-10.

Associate editor: Federico Hoffmann 